\title{
Impact des médicaments sur l'environnement : état des lieux, évaluation des risques, communication
}

\author{
Thierry Moreau Defarges ${ }^{1}$, Michel Guerbet ${ }^{2}$, Jacques Massol ${ }^{3}$ et les participants à la Table Ronde $N^{\circ} 4$ \\ de Giens XXVI ${ }^{\star}$ \\ 1 Cyclamed, Boulogne-Billancourt, France \\ 2 Université de Rouen, UFR Médecine Pharmacie, ADEN EA 4311, Rouen, France \\ 3 Hôpital Saint-Jacques, Besançon, France
}

Texte reçu le 15 mars 2011 ; accepté le 30 mai 2011

\begin{abstract}
Mots clés :
Cyclamed ;

médicaments

non utilisés ;

évaluation du risque ;

santé ;

pollution ;

environnement

Résumé - L'objet de cette Table Ronde était de faire un premier état des lieux sur l'impact des médicaments sur l'environnement. La croissance démographique dans le monde, la demande de santé de plus en plus forte, la consommation de médicaments qui ne cesse de se développer, conduisent à une présence de plus en plus importante des substances médicamenteuses dans les différents compartiments (air, eau, sol) avec des répercussions potentielles sur l'environnement et la santé. Pour la première fois les Ateliers de Pharmacologie de Giens ont programmé ce thème en dehors du champ d'action classique de Giens. Un intérêt très important pour ce thème s'est dégagé tant des membres de la Table Ronde que des auditeurs, et il est certain que celle-ci ouvre la porte à de nouvelles initiatives pour un sujet encore mal connu. Ont donc été abordés successivement : l'éclatement des connaissances sur le sujet, et leurs insuffisances concernant l'impact sanitaire aussi bien des rejets dans leur globalité que de certains médicaments particuliers, les performances des méthodes de traitement des eaux, les sources de pollution, l'impact environnemental et l'impact sanitaire, les pistes d'évolution règlementaires et les formations à mettre en place auprès de tous les acteurs professionnel et grand public.
\end{abstract}

\section{Introduction}

La présence de résidus médicamenteux dans les milieux aquatiques a été détectée dans les années 1980 et a conduit les scientifiques à s'interroger sur les conséquences sur l'environnement et la santé humaine de ces polluants. ${ }^{[1,2]}$ Plusieurs milliers de tonnes de médicaments à usage humain ou vétérinaire sont en effet utilisés chaque année dans le monde et rejetés, en nature ou après métabolisation, dans l'environnement. La contamination environnementale par des résidus de médicaments est aujourd'hui clairement démontrée par de nombreuses études de terrain et font des rejets médicamenteux un problème environnemental émergent. ${ }^{[2-6]}$

Des résidus médicamenteux ont été retrouvés dans les eaux superficielles, eaux souterraines, eaux résiduaires, boues de sta-

\footnotetext{
^ Pour la liste des participants, voir en fin d'article
}

tion d'épuration mais aussi dans certains échantillons d'eau de boisson.

Par rapport aux nombreux autres polluants environnementaux (pesticides, métaux lourds, hydrocarbures...), le risque sanitaire redouté est plus particulièrement lié à la spécificité des médicaments qui sont des substances présentant une forte activité biologique à de faibles concentrations. Si certains effets sur la faune et la flore sont aujourd'hui assez bien connus ${ }^{[5,7]}$ (résistance bactérienne liée aux antibiotiques; effets perturbateurs endocriniens des hormones et molécules apparentées; génotoxicité de nombreux médicaments anticancéreux...), les conséquences sanitaires de la pollution environnementale par les rejets de médicaments présentent encore de nombreuses inconnues ${ }^{[8-10]}$ et les méthodes pour les mettre en évidence sont difficiles, non standardisées et peu développées.

La complexité du problème est par ailleurs aggravée par la très grande diversité de molécules utilisées, rejetés 


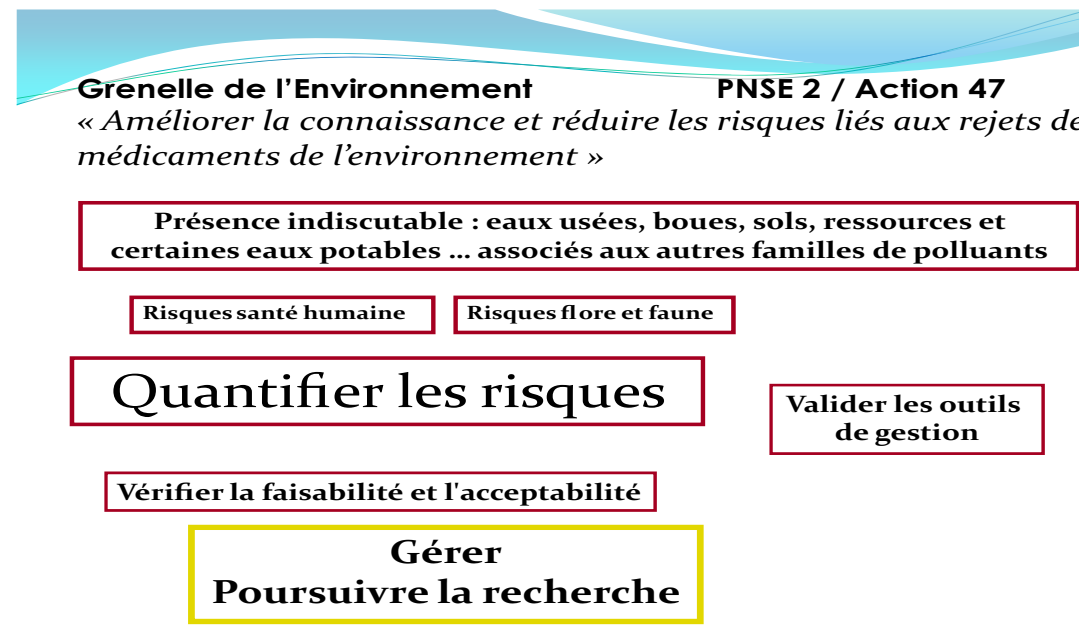

Fig. 1. Le médicament dans le Grenelle de l'environnement.

essentiellement par voie fécale ou urinaire. Les sources de dissémination sont nombreuses, à la fois diffuses (population générale traitée à domicile) et ponctuelles (industrie pharmaceutique, établissements de soins). ${ }^{[11]}$

Dans la majorité des cas, les résidus médicamenteux sont éliminés dans les réseaux d'eaux usées urbaines pour aboutir aux stations d'épuration des eaux usées (STEP) qui, par définition, ne sont pas conçues pour éliminer les médicaments, et dont l'efficacité est extrêmement variable, entre $0 \%$ pour certains composés et plus de $90 \%$ pour d'autres molécules. ${ }^{[12]}$

Le Comité d'Organisation de Giens a pris conscience de ces enjeux et a inscrit ce thème méconnu au sein des Ateliers de Giens 2010, thème que nous avons eu le privilège de développer avec des représentants des Autorités, des industriels et des experts.

Cette Table Ronde s'inscrit à la fois dans le cadre du Grenelle de l'Environnement et dans le cadre du Plan national résidus de médicaments (PNRM) [figure 1].

Le sujet des résidus médicamenteux est un sujet à la fois vaste et complexe. C'est la raison pour laquelle, dans le cadre des Ateliers de Giens, il a été choisi de limiter le champ de réflexions à certains aspects jugés prioritaires et/ou plus directement en relation avec les compétences des participants à cette Table Ronde.

Bien que les différents compartiments de l'environnement puissent être concernés par les résidus médicamenteux, c'est le compartiment hydrique qui est celui dans lequel la contamination est la plus à redouter et qui a fait l'objet du plus grand nombre de travaux scientifiques. Nous nous sommes donc limités au compartiment « eau ».

Par ailleurs, les médicaments à usage humain ont été retenus comme source prioritaire de pollution prenant en compte ainsi la qualité des participants de Giens concentrés sur la médecine humaine (le médicament vétérinaire doit faire l'objet d'études sé- parées et complémentaires compte tenu des quantités massives que ces produits représentent). De même, les problèmes spécifiques liés aux radionucléides n'ont pas été abordés.

Nous avons souhaité que les réflexions sur ce sujet ne se limitent pas au risque environnemental pour la flore et la faune et prennent en considération l'impact sanitaire direct ou indirect des rejets de médicaments dans l'environnement.

Enfin, tous les participants ont considéré que, en préambule à toute réflexion sur les risques de contamination environnementale par les résidus médicamenteux, il est important de rappeler l'intérêt thérapeutique des médicaments et leur rapport bénéfice/risque.

Quatre questions ont donc été posées :

- Quelles connaissances doivent être améliorées ? Et comment?

- Comment réduire les sources?

- Comment faire évoluer la prise en compte du risque environnemental dans le dossier d'autorisation de mise sur le marché (AMM) et le dossier de transparence?

- Quelle communication adopter? Comment sensibiliser, former les citoyens?

\section{Quelles connaissances à améliorer et comment?}

En ce début de $\mathrm{XXI}^{\mathrm{e}}$ siècle, on ne peut que constater que nos connaissances dans ce domaine restent très parcellaires, limitées. Si l'on a quelques données sur des molécules spécifiques et leurs conséquences sur la santé humaine, il reste que les études d'impact sur celle-ci sont encore extrêmement limitées et que leur méthodologie mérite d'être développée. Les effets des résidus de médicaments sur l'homme sont encore à démontrer bien que l'on ait déjà pu constater des conséquences sur la faune. 


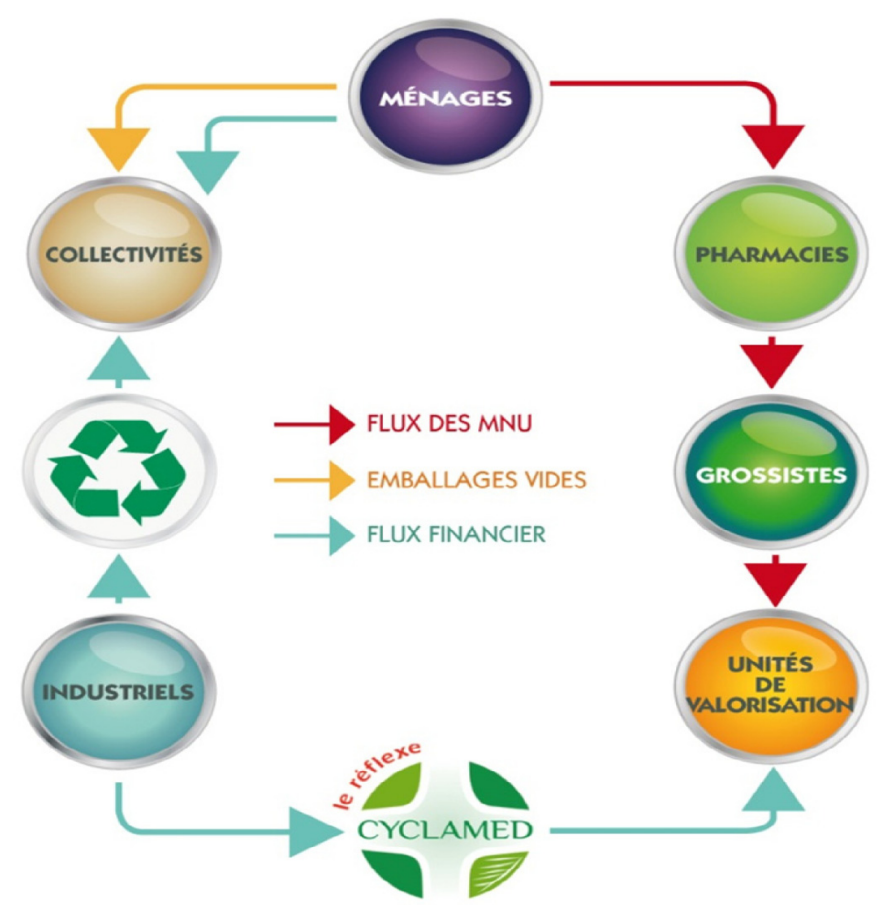

Fig. 2. Rôle du dispositif Cyclamed pour réduire l'impact environnemental du médicament (avec l'aimable autorisation de Cyclamed).

Parmi les points à examiner de manière approfondie, on peut citer :

- les effets sur la santé humaine de faibles concentrations à long terme;

- l'impact sanitaire global des milieux (en premier lieu hydrique) contenant des médicaments en prenant compte des interactions médicamenteuses et non médicamenteuses avec d'autres polluants (ne pas oublier que le médicament n'est pas un polluant isolé);

- les effets des mélanges de médicaments ou de médicaments et d'autres substances consommées régulièrement par les humains ;

- les effets sur des populations sensibles ou spécifiques (femmes enceintes, enfants, personnes âgées etc....);

- le rôle des produits de transformation;

- l'amélioration des systèmes de traitement des eaux usées domestiques et hospitalières.

\section{Comment réduire les sources?}

Quatre sources principales d'émission dans l'environnement de médicaments à usage humain ont été identifiées :

- l'excrétion par les patients par les urines ou les fèces, soit sous forme originelle active, soit sous forme de métabolite actif ou inactif;
- les rejets industriels involontaires ou volontaires, accidentels ou non par les sites de synthèses chimiques ou de production;

- les rejets de médicaments non utilisés (MNU) par les patients qui, au lieu de rapporter ceux-ci en officine, les jettent soit dans leur poubelle soit dans leur toilettes ou les dissolvent avec de l'eau du robinet;

- les rejets par les laboratoires universitaires, de recherche ou de biologie ou même par les établissements de soins (hôpitaux, cliniques) ou par le personnel soignant ambulatoire.

Il est donc nécessaire de mettre en place sur chacune de ces sources, des mesures adéquates pour limiter la contamination de notre environnement. Deux domaines ont déjà fait l'objet de programmes très importants :

\section{- Les rejets industriels}

L'industrie pharmaceutique et chimique a pris, depuis des accidents des années 1980, des mesures drastiques pour limiter les risques de rejets accidentels en mettant par exemple en place des cuves de récupération pour les eaux ayant servi à éteindre un incendie.

\section{- Les médicaments non utilisés}

La France a été le premier pays en Europe en 1993-1994 à mettre en place un système de récupération et de valorisation 
énergétique des MNU par les ménages (système Cylamed). Le pharmacien a l'obligation de récupérer ceux-ci qui sont ensuite transportés par les grossistes dans leur centre de distribution puis vers des unités de valorisation énergétique (figure 2). C'est ainsi qu'environ 13500 tonnes de MNU sont ainsi valorisées chaque année. ${ }^{[1]}$

\section{Pourquoi et comment faire évoluer la prise en compte du risque environnemental dans le dossier d'AMM et le dossier de transparence?}

Face à l'importance grandissante du risque environnemental, il apparaît nécessaire de faire évoluer les dossiers qui permettent l'autorisation de mise sur le marché des spécialités pharmaceutiques de même que ceux fournis en vue de la demande d'admission au remboursement.

Au même titre que l'évaluation de l'efficacité et de la tolérance, l'impact environnemental et ses conséquences sanitaires devront, à l'avenir, être pris en compte par les décideurs publics ; aussi importe-t-il, pour ce faire, de leur apporter un éclairage scientifique.

L'écotoxicité des nouveaux médicaments est importante à connaître au moment de l'AMM non pour en empêcher l'autorisation qui repose sur le bénéfice et le risque pour le patient mais pour chercher, le cas échéant, à limiter voire à antagoniser cette toxicité et un éventuel impact négatif du médicament sur l'environnement. La demande d'études d'écopharmacoépidémiologie dans le cadre des études post-AMM réglementaires par exemple par un volet environnemental des plans de gestion de risque est une opportunité d'améliorer l'état des connaissances sur le sujet.

Depuis 2006, l'impact environnemental est pris en compte dans le dossier d'AMM pour les produits nouveaux, mais les données restent confidentielles, ce qui constitue un facteur limitant pour certaines études de terrain. Par ailleurs, cette évaluation se heurte encore à différentes difficultés méthodologiques. Le programme Knowledge and need assessment on pharmaceuticals products in environnement waters (KNAPPE) ${ }^{[13]}$ a porté entre 2005 et 2009 sur l'identification des actions prioritaires à mener pour limiter l'impact des produits pharmaceutiques dans l'environnement. Il a relevé le manque de tests normalisés pour évaluer certains effets, comme les effets perturbateurs endocriniens, et la nécessité d'initier de nouveaux programmes européens sur ce sujet de l'évaluation des effets.

Dans le cadre de l'admission en remboursement, si l'on peut envisager l'idée qu'un impact environnemental puisse modifier le service médical rendu par le médicament à la collectivité, et qu'à performances médicales comparables un médicament plus respectueux de l'environnement soit susceptible de rendre un meilleur service, il n'existe pas, à ce jour de critère explicite prenant en compte l'impact du médicament sur l'environnement. Les participants de la Table Ronde étaient d'accord sur la nécessité d'apporter un éclairage scientifique aux décideurs publics sur l'impact environnemental attendu du médicament mais ni la structure impliquée dans cette évaluation (nécessairement pluridisciplinaire), ni le critère à utiliser [intégration dans un intérêt de santé public (ISP) modifié] n'a fait consensus.

De nouveaux critères, tels que l'éco-conception du médicament ou la mise à disposition d'antagonistes ou de systèmes de neutralisation des rejets médicamenteux ne devraient-ils pas être incorporés dans la décision de fixation du prix?

Une majorité d'acteurs considère aujourd'hui que le médicament doit être mis au même niveau que les autres produits (biocides, ...) et que de ce fait les lignes directrices précisant les règles d'évaluation du risque environnemental de tous ces composés devraient être révisées dans le sens d'une harmonisation des critères d'évaluation. Pour les environnementalistes, le médicament humain doit rentrer dans le droit commun vis-à-vis de l'environnement. Si on ne peut être, en tant que citoyen que d'accord sur ce principe, il convient néanmoins de prendre en compte le rôle que le médicament apporte à la santé publique et qu'il doit faire l'objet d'une règlementation adaptée.

\section{Comment communiquer, comment sensibiliser?}

La communication autour du risque environnemental et du risque sanitaire reste particulièrement complexe et sensible pour les populations. Il convient d'informer sans paniquer et de trouver « un juste équilibre entre alarme et négligence». Il apparaît donc important de différencier des types de communication par catégories de citoyens :

- pour le grand public, la communication doit s'orienter dans un premier temps sur des actions préventives que chacun peut et doit mettre en place comme le tri sélectif, le retour des MNU en officine, le respect de certains gestes pour limiter la contamination des milieux naturels;

- pour les professionnels de santé, une meilleure information sur le problème des rejets médicamenteux dans l'environnement paraît indispensable tout en leur demandant également d'être des relais auprès de leur patient sur les actions préventives qu'ils peuvent mener.

Enfin, la nouvelle organisation de notre système de santé, par la loi HPST, ${ }^{[14]}$ donne un rôle particulier aux Agences régionales de santé dont le périmètre d'action couvre largement les problèmes 
de santé et d'environnement. Il y a là sûrement un point d'appui majeur pour la communication dans les années qui viennent.

\section{Synthèse des recommandations}

Dans ce domaine complexe des rejets de médicaments dans l'environnement, les acteurs sont nombreux, du producteur au consommateur, et on doit reconnaître que des efforts ont été accomplis par chacun depuis plusieurs années. Il reste que la prise de conscience du problème est encore récente et que des mesures doivent être prises pour anticiper les conséquences à long terme sur notre environnement, sur notre santé et la santé des générations futures.

En pratique, sur chacune des questions posées, nous proposons les quelques thèmes de travail.

\section{1. Connaissances à améliorer}

- Cibler des actions spécifiques dans les programmes de recherche fondamentale sur ces risques à plus ou moins long terme pour l'homme.

- Élaborer et/ou adapter les méthodes épidémiologiques pour étudier l'impact sanitaire des médicaments sur l'environnement.

- Acquérir des données éco-pharmaco-épidémiologiques d'exposition et d'effets.

- Encourager les partenariats public/privé dans ce secteur.

- Apprécier les performances des filières de traitement de l'eau sur des molécules prioritaires.

- Focaliser la recherche sur des familles de molécules prioritaires (produits à activité hormonale, cytotoxiques, antibiotiques...).

- Développer l'information sur les médicaments issus des nouvelles technologies (les nano-médicaments) et biothérapies.

\subsection{Réduction des sources}

- Évaluer la pertinence d'un prétraitement à la source des effluents hospitaliers et des établissements médico-sociaux.

- Encourager la recherche de produits de neutralisation pour les molécules les plus écotoxiques.

- Sensibiliser les laboratoires de recherche et leurs tutelles.

- Optimiser les traitements des rejets de l'industrie chimique et pharmaceutique.

\section{3. Évolution de la prise en compte du risque environnemental dans l'évaluation scientifique (dossier d'AMM, transparence) et dans les décisions publiques (AMM, inscription au remboursement, prix)}

- Demander des études d'impact environnemental et sanitaire pour une liste prioritaire de molécules « anciennes » non documentées car mises sur le marché avant 2006 (date d'exigence réglementaire de réaliser une évaluation du risque environnemental dans le dossier d'AMM) mais présentant un risque suspecté (hormones, cytotoxiques, antibiotiques...) ou étant des molécules de grande consommation.

- Introduire un volet environnemental au sein du plan de gestion des risques (PGR).

- Évaluer l'impact direct ou indirect sur la santé de l'homme lié aux résidus de médicaments et autres produits de santé. Faut-il intégrer cette donnée dans un nouvel intérêt de santé publique? Le débat est ouvert.

- Mettre en place d'un éco-bonus et/ou d'un éco-malus.

- Introduire la dimension de développement durable dans l'accord cadre entre les entreprises du médicament (LEEM) et le Comité économique des produits de santé (CEPS).

\subsection{Comment communiquer?}

- Poursuivre et amplifier les campagnes de sensibilisation du grand public au retour des MNU à l'officine (Cyclamed).

- Sensibiliser les professionnels de santé, les étudiants des professions médicales et paramédicales sur le problème des résidus de substances médicamenteuses dans l'environnement.

- Développer la notion de tri sélectif dans l'esprit du citoyen.

\section{Conclusion}

Toutes ces propositions doivent être prises en compte comme des pistes de travail. En effet, leur coût et leur réalisation pratique doivent être clairement appréhendés au regard du bénéfice qu'apportent les médicaments à l'homme. Par ailleurs, cette problématique doit également prendre en considération la médecine vétérinaire, grosse consommatrice de médicaments avec des rejets importants (lisiers, ... ), et s'intégrer dans une approche plus globale de pollution environnementale dans laquelle le médicament n'est qu'un des facteurs d'un contexte multifactoriel dans lequel sont impliquées de nombreuses catégories de contaminants chimiques (produits de nettoyage, biocides, pesticides, etc.).

Les médicaments et l'environnement, un chantier clé pour le $\mathrm{XXI}^{\mathrm{e}}$ siècle. 


\section{Participants}

Jacques Aumonier (Cephalon), Nathalie Billon (Sanofi-Aventis), Claude Casellas (Université Montpellier), Chantal Gatignol (DGS), Michel Guerbet (Université Rouen), Paul Houeto (Afssaps), Romain Journel (Sanofi-Aventis), Hervé Le Louet (AP-HP), Benoit Lesaffre (DGS), Jacques Massol (DGS), Thierry Moreau Defarges (Cyclamed), Anne Pham Ba (DGS), Claire Sibenaler (LEEM).

\section{Références}

1. Cyclamed. Rapport d'activité 2009. http://www. cyclamed.org

2. Fatta-Kassinos D, Meric S, Nikolaou A. Pharmaceuticals residues in environmental waters and wastewater: current state of knowledge and future research. Anal Bioanal Chem 2011; 399: 251-75

3. Bottoni P, Caroli S, Barra Caracciolo A. Pharmaceuticals as priority water contaminants. Tox Environ Chem 2010; 92(3): 549-65

4. Fenet H, Gomez E, Leclerc M, et al. Devenir des médicaments dans l'environnement. Environnement, Risques et Santé 2006; 5(4): 243-7

5. Kent K, Weston AA, Caminada D. Ecotoxicology of human pharmaceuticals. Aquat Tox 2006; 76: 122-59

6. Leibig M, Moltmann JF, Knacker T. Evaluation of measured and predicted environmental concentrations of selected pharmaceuticals and personal care products. Environ Sci \& Pollut Res 2006; 13(2): 110-9
7. Pal A, Yew-Hoong Gin $\mathrm{K}$, Yu-Chen Li A, et al. Impacts of emerging or ganic contaminants on freshwater resources: review of recent occurrences, sources, fate and effects. Sci Tot Environ 2010; 408: 6062-9

8. Garric J, Ferrari B. Les substances pharmaceutiques dans les milieux aquatiques. Niveaux d'exposition et effet biologique. Rev Sci Eau 2005; 18(3): 307-30

9. Haguenoer JM. Médicaments et eaux. Rapport de l'Académie Nationale de Pharmacie 2008; 105 p. http: //www . acadpharm.org

10. Prescrire. La pollution des eaux par le médicament. La Revue Prescrire 2007 27(284): 460-4

11. Daughton CG, Ternes TA. Pharmaceuticals and personal care products in the environment: agents of subtle change? Environ Health Perspect 1999; 107(6): 907-42

12. Castiglioni S, Bagnati R, Fanelli R, et al. Removal of pharmaceuticals in sewage tratment plants in Italy. Environ Sci Technol 2006; 40: 357-63

13. http://www.knappe-eu.org

14. Journal Officiel. 22 juillet 2009 JORF $n^{\circ} 0167$ p 12184. Texte $n^{\circ} 1$. Loi $\mathrm{N}^{\circ}$ 2009-879 du 21 juillet portant réforme de l'hôpital et relative aux patients, à la santé et aux territoires. http://www. legifrance.gouv. fr

Correspondance et offprints : Thierry Moreau Defarges, Cylcamed, 86 rue Thiers, 92100 Boulogne, France.

E-mail : tnmd.cyclamed@orange.fr 